\title{
On Generalized Padovan Numbers
}

\author{
Yüksel Soykan \\ Department of Mathematics, Art and Science Faculty, \\ Zonguldak Bülent Ecevit University, 67100, Zonguldak, Turkey \\ e-mail: yuksel_soykan@hotmail.com
}

\begin{abstract}
In this paper, we investigate the generalized Padovan sequences and we deal with, in detail, four special cases, namely, Padovan, Perrin, Padovan-Perrin and modified Padovan sequences. We present Binet's formulas, generating functions, Simson formulas, and the summation formulas for these sequences. Moreover, we give some identities and matrices related with these sequences.
\end{abstract}

2010 Mathematics Subject Classification. 11B39, 11B83.

Keywords. Padavon numbers, Perrin numbers, Padovan-Perrin numbers.

\section{Introduction}

Recently, there have been so many studies of the sequences of numbers in the literature that concern about subsequences of the Horadam numbers and generalized Tribonacci numbers such as Fibonacci, Lucas, Pell and Jacobsthal numbers; Tribonacci, Tribonacci-Lucas, Narayana, third order Jacobsthal and third order Jacobsthal-Lucas numbers. The sequences of numbers were widely used in many research areas, such as physics, engineering, architecture, nature and art. The ratio of two consecutive Fibonacci numbers converges to the Golden section (ratio), $\alpha_{F}=\frac{1+\sqrt{5}}{2}$; which appears in modern research, particularly physics of the high energy particles or theoretical physics. Another example, the ratio of two consecutive Tribonacci numbers converges to the Tribonacci ratio, $\alpha_{T}=\frac{1+\sqrt[3]{19+3 \sqrt{33}}+\sqrt[3]{19-3 \sqrt{33}}}{3}$. One last example, the ratio of two consecutive Padovan numbers converges to the Plastic ratio, $\alpha_{P}$ (which is given in (1.1) below), which have many applications to such as architecture, see [53]. For a short introduction to these three constants, see [62]. 
Padovan (Cordonnier) numbers, Perrin (Padovan-Lucas) numbers and Van der Laan numbers are defined, respectively, by the third-order recurrence relations

$$
\begin{aligned}
& P_{n+3}=P_{n+1}+P_{n}, \quad P_{0}=1, P_{1}=1, P_{2}=1, \\
& E_{n+3}=E_{n+1}+E_{n}, \quad E_{0}=3, E_{1}=0, E_{2}=2, \\
& R_{n+3}=R_{n+1}+R_{n}, \quad R_{0}=1, R_{1}=0, R_{2}=1, \text { or } R_{0}=0, R_{1}=1, R_{2}=0 .
\end{aligned}
$$

For historical background issues on these particular cases of generalized Padovan sequences, see [68].

Edouard Lucas [51] in 1876 introduced the sequence $E_{n}$ (Perrin sequence, see for example [56]) and the sequence $E_{n}$ was also discussed by Lucas in 1878 (American Journal of Mathematics, vol 1, page 230ff), who noted that if $p$ is a prime then $p$ divides $E_{n}$. This is an immediate consequence of Fermat's Little Theorem, and as such is a necessary but not sufficient condition for primality (for a proof see [57]). Subsequently (1899) the same sequence was mentioned by R. Perrin [60]. There are some other papers on the sequence $E_{n}$ after Perrin's works, see [26,40,52]. The most extensive (published) treatment of this sequence was given in an excellent paper by Bill Adams and Dan Shanks in [2]. Shanks and Adams referred (called) to this as Perrin's sequence.

Originating and naming of $\left\{P_{n}\right\}$ is rather less straightforward. Termed by Steward [80] (and Broadhurst and Kreimer [9] with the initial condion $P_{0}=0, P_{1}=0, P_{2}=1$ ) as the Padovan numbers in honour of the contemporary architect Richard Padovan, these numbers seemingly have a more extensive origine. The sequence $\left\{P_{n}\right\}$ seems to have been first discovered in 1924 by a French architecture student, Gérard Cordonnier and independently, the $\left\{P_{n}\right\}$ were rediscovered by Dom Hans van der Laan (see [68]).

The characteristic equation associated with Padovan, Perrin, Van der Laan sequences is $x^{3}-x-1=0$ with roots $\alpha, \beta$ and $\gamma$ in which

$$
\alpha=\left(\frac{1}{2}+\sqrt{\frac{23}{108}}\right)^{1 / 3}+\left(\frac{1}{2}-\sqrt{\frac{23}{108}}\right)^{1 / 3} \simeq 1.32471795724
$$

is called plastic number (or plastic ratio or plastic constant or silver number) and

$$
\lim _{n \rightarrow \infty} \frac{P_{n+1}}{P_{n}}=\lim _{n \rightarrow \infty} \frac{E_{n+1}}{E_{n}}=\alpha .
$$

The plastic number is used in art and architecture. Richard Padovan studied on plastic number in Architecture and Mathematics in [58, 59]. The "plastic number" made popular by Richard Padovan. Padovan pointed out that the plastic number was invented by a French architectural student, Gérard Cordonnier, in 1924 and by a Dutch Benedictine monk-architect, Hans van der Laan, in 1928. Pastic number was originally studied by G. Cordonnier in 1924. However, Hans van der Laan was the first who explained how it relates to the human perception of differences in size between three-dimensional objects and demonstrated his discovery in architectural design. Laan's main premise was that the plastic number ratio is "truly aesthetic in the original Greek sense, i.e. that its concern is not 'beauty' but clarity of perception" (see [58]). Cordonnier described applications to architecture, using the name radiant number and in 1958 he gave a lecture tour 
that illustrated the use of the plastic number in many existing buildings and monuments. Marohnić and Strmećki [53] constructed the Plastic number in a heuristic way, explaining its relation to human perception in three-dimensional space through architectural style of Dom Hans van der Laan. Note that plastic number is a morphic number, see [1] for details. For more details on plastic number, see $[5,38,79,85,87]$.

Recently, these sequences $\left(\left\{P_{n}\right\},\left\{E_{n}\right\},\left\{R_{n}\right\}\right)$ have been studied extensively by many authors, see for instance $[3,8,15,36,27,46,49,66,69,73,74,80,86,89]$. See also web pages [ $54,55,56]$ for Padovan numbers.

Kaygisiz and Bozkurt [43] defined k sequences of generalized order-k Perrrin numbers. Kaygisiz and Sahin [44] defined generalized Van der Laan and Perrin Polynomials, and generalizations of Van der Laan and Perrin Numbers.

Many researchers have studied matrix representations of number sequences. In [92] and [94], Yilmaz and Taskara developed the matrix sequences that represent Padovan and Perrin numbers. Şahin [64] defined and studied generalized Perrin and Cordonnier matrices using the associated polynomials of Perrin and Cordonnier numbers. Kaygisiz and Sahin [45] calculated terms of associated polynomials of Perrin and Cordonnier numbers by using determinants and permanents of various Hessenberg matrices. In [90] authors gave matrix representation of Perrin sequences. See also [66,73,74,72] for Padovan Q-matrix and related matrices. In [16], Cereceda provided some determinantal representation of the Padovan numbers by using the Hessenberg matrices.

The Padovan numbers and their properties have been studied by some other authors too, see for example, $[4,6,10,18,19,21,22,23,28,29,30,31,32,33,34,35,39,42,63,81,82,84,91,93,96]$

It is the aim of this paper to define and to explore some of the properties of generalized Padovan numbers and is to investigate, in details, four particular case, namely sequences of Padovan, Perrin, Padovan-Perrin and modified Padovan numbers $\left\{P_{n}\right\},\left\{E_{n}\right\},\left\{S_{n}\right\}$ and $\left\{A_{n}\right\}$, respectively. Before, we recall the generalized Tribonacci sequence and its some properties.

The generalized Tribonacci sequence $\left\{W_{n}\left(W_{0}, W_{1}, W_{2} ; r, s, t\right)\right\}_{n \geq 0}$ (or shortly $\left.\left\{W_{n}\right\}_{n \geq 0}\right)$ is defined as follows:

$$
W_{n}=r W_{n-1}+s W_{n-2}+t W_{n-3}, \quad W_{0}=a, W_{1}=b, W_{2}=c, \quad n \geq 3
$$

where $W_{0}, W_{1}, W_{2}$ are arbitrary complex (or real) numbers and $r, s, t$ are real numbers.

This sequence has been studied by many authors, see for example $[11,12,17,24,25,50,61,65,67,77,78,88,95]$.

The sequence $\left\{W_{n}\right\}_{n \geq 0}$ can be extended to negative subscripts by defining

$$
W_{-n}=-\frac{s}{t} W_{-(n-1)}-\frac{r}{t} W_{-(n-2)}+\frac{1}{t} W_{-(n-3)}
$$

for $n=1,2,3, \ldots$ when $t \neq 0$. Therefore, recurrence (1.2) holds for all integer $n$.

As $\left\{W_{n}\right\}$ is a third order recurrence sequence (difference equation), it's characteristic equation is

$$
x^{3}-r x^{2}-s x-t=0
$$


whose roots are

$$
\begin{aligned}
& \alpha=\alpha(r, s, t)=\frac{r}{3}+A+B \\
& \beta=\beta(r, s, t)=\frac{r}{3}+\omega A+\omega^{2} B \\
& \gamma=\gamma(r, s, t)=\frac{r}{3}+\omega^{2} A+\omega B
\end{aligned}
$$

where

$$
\begin{aligned}
& A=\left(\frac{r^{3}}{27}+\frac{r s}{6}+\frac{t}{2}+\sqrt{\Delta}\right)^{1 / 3}, B=\left(\frac{r^{3}}{27}+\frac{r s}{6}+\frac{t}{2}-\sqrt{\Delta}\right)^{1 / 3} \\
& \Delta=\Delta(r, s, t)=\frac{r^{3} t}{27}-\frac{r^{2} s^{2}}{108}+\frac{r s t}{6}-\frac{s^{3}}{27}+\frac{t^{2}}{4}, \omega=\frac{-1+i \sqrt{3}}{2}=\exp (2 \pi i / 3)
\end{aligned}
$$

Note that we have the following identities

$$
\begin{aligned}
\alpha+\beta+\gamma & =r, \\
\alpha \beta+\alpha \gamma+\beta \gamma & =-s, \\
\alpha \beta \gamma & =t .
\end{aligned}
$$

If $\Delta(r, s, t)>0$, then the Equ. (1.3) has one real $(\alpha)$ and two non-real solutions with the latter being conjugate complex. So, in this case, it is well known that generalized Tribonacci numbers can be expressed, for all integers $n$, using Binet's formula

$$
W_{n}=\frac{b_{1} \alpha^{n}}{(\alpha-\beta)(\alpha-\gamma)}+\frac{b_{2} \beta^{n}}{(\beta-\alpha)(\beta-\gamma)}+\frac{b_{3} \gamma^{n}}{(\gamma-\alpha)(\gamma-\beta)}
$$

where

$$
b_{1}=W_{2}-(\beta+\gamma) W_{1}+\beta \gamma W_{0}, b_{2}=W_{2}-(\alpha+\gamma) W_{1}+\alpha \gamma W_{0}, b_{3}=W_{2}-(\alpha+\beta) W_{1}+\alpha \beta W_{0} .
$$

Note that the Binet form of a sequence satisfying (1.3) for non-negative integers is valid for all integers $n$, for a proof of this result see [37]. This result of Howard and Saidak [37] is even true in the case of higher-order recurrence relations.

In this paper we consider the case $r=0, s=t=1$ and in this case we write $V_{n}=W_{n}$. A generalized Padovan sequence $\left\{V_{n}\right\}_{n \geq 0}=\left\{V_{n}\left(V_{0}, V_{1}, V_{2}\right)\right\}_{n \geq 0}$ is defined by the third-order recurrence relations

$$
V_{n}=V_{n-2}+V_{n-3}
$$

with the initial values $V_{0}=c_{0}, V_{1}=c_{1}, V_{2}=c_{2}$ not all being zero.

The sequence $\left\{V_{n}\right\}_{n \geq 0}$ can be extended to negative subscripts by defining

$$
V_{-n}=-V_{-(n-1)}+V_{-(n-3)}
$$

for $n=1,2,3, \ldots$. Therefore, recurrence (1.5) holds for all integer $n$. 
(1.4) can be used to obtain Binet formula of generalized Padovan numbers. Binet formula of generalized padovan numbers can be given as

$$
V_{n}=\frac{b_{1} \alpha^{n}}{(\alpha-\beta)(\alpha-\gamma)}+\frac{b_{2} \beta^{n}}{(\beta-\alpha)(\beta-\gamma)}+\frac{b_{3} \gamma^{n}}{(\gamma-\alpha)(\gamma-\beta)}
$$

where

$$
b_{1}=V_{2}-(\beta+\gamma) V_{1}+\beta \gamma V_{0}, b_{2}=V_{2}-(\alpha+\gamma) V_{1}+\alpha \gamma V_{0}, b_{3}=V_{2}-(\alpha+\beta) V_{1}+\alpha \beta V_{0}
$$

Here, $\alpha, \beta$ and $\gamma$ are the roots of the cubic equation $x^{3}-x-1=0$. Moreover

$$
\begin{aligned}
& \alpha=\left(\frac{1}{2}+\sqrt{\frac{23}{108}}\right)^{1 / 3}+\left(\frac{1}{2}-\sqrt{\frac{23}{108}}\right)^{1 / 3}=1.32471795724 \\
& \beta=\omega\left(\frac{1}{2}+\sqrt{\frac{23}{108}}\right)^{1 / 3}+\omega^{2}\left(\frac{1}{2}-\sqrt{\frac{23}{108}}\right)^{1 / 3} \\
& \gamma=\omega^{2}\left(\frac{1}{2}+\sqrt{\frac{23}{108}}\right)^{1 / 3}+\omega\left(\frac{1}{2}-\sqrt{\frac{23}{108}}\right)^{1 / 3}
\end{aligned}
$$

where

$$
\omega=\frac{-1+i \sqrt{3}}{2}=\exp (2 \pi i / 3)
$$

Note that

$$
\begin{aligned}
\alpha+\beta+\gamma & =0, \\
\alpha \beta+\alpha \gamma+\beta \gamma & =-1, \\
\alpha \beta \gamma & =1 .
\end{aligned}
$$

The first few generalized Padovan numbers with positive subscript and negative subscript are given in the following Table 1.

Table 1. A few generalized Padovan numbers

\begin{tabular}{ccc}
\hline$n$ & $V_{n}$ & $V_{-n}$ \\
\hline 0 & $V_{0}$ & $\cdots$ \\
1 & $V_{1}$ & $V_{2}-V_{0}$ \\
2 & $V_{2}$ & $-V_{2}+V_{1}+V_{0}$ \\
3 & $V_{1}+V_{0}$ & $V_{2}-V_{1}$ \\
4 & $V_{2}+V_{1}$ & $V_{1}-V_{0}$ \\
5 & $V_{2}+V_{1}+V_{0}$ & $-V_{2}+2 V_{0}$ \\
6 & $V_{2}+2 V_{1}+V_{0}$ & $2 V_{2}-V_{1}-2 V_{0}$ \\
7 & $2 V_{2}+2 V_{1}+V_{0}$ & $-2 V_{2}+2 V_{1}+V_{0}$ \\
8 & $2 V_{2}+3 V_{1}+2 V_{0}$ & $V_{2}-2 V_{1}+V_{0}$ \\
\hline
\end{tabular}


Now we define four special cases of the sequence $\left\{V_{n}\right\}$. Padovan (Cordonnier) sequence $\left\{P_{n}\right\}_{n \geq 0}$, Perrin (Padovan-Lucas) sequence $\left\{E_{n}\right\}_{n \geq 0}$, Padovan-Perrin sequence $\left\{S_{n}\right\}_{n \geq 0}$ and modified Padovan sequence $\left\{A_{n}\right\}_{n \geq 0}$ are defined, respectively, by the third-order recurrence relations

$$
\begin{array}{ll}
P_{n+3}=P_{n+1}+P_{n}, & P_{0}=1, P_{1}=1, P_{2}=1, \\
E_{n+3}=E_{n+1}+E_{n}, & E_{0}=3, E_{1}=0, E_{2}=2, \\
S_{n+3}=S_{n+1}+S_{n}, & S_{0}=0, S_{1}=0, S_{2}=1, \\
A_{n+3}=A_{n+1}+A_{n}, & A_{0}=3, A_{1}=1, A_{2}=3 .
\end{array}
$$

Note that the case $V_{n}=R_{n}, R_{0}=1, R_{1}=0, R_{2}=1$ (or $V_{n}=R_{n}, R_{0}=0, R_{1}=1, R_{2}=0$ ) is called the sequence of the Van der Laan numbers, in the literature.

The sequences $\left\{P_{n}\right\}_{n \geq 0},\left\{E_{n}\right\}_{n \geq 0},\left\{S_{n}\right\}_{n \geq 0}$ and $\left\{A_{n}\right\}_{n \geq 0}$ can be extended to negative subscripts by defining

$$
\begin{aligned}
& P_{-n}=-P_{-(n-1)}+P_{-(n-3)} \\
& E_{-n}=-E_{-(n-1)}+E_{-(n-3)} \\
& S_{-n}=-S_{-(n-1)}+S_{-(n-3)} \\
& A_{-n}=-A_{-(n-1)}+A_{-(n-3)}
\end{aligned}
$$

for $n=1,2,3, \ldots$ respectively. Therefore, recurrences $(1.7),(1.8),(1.9)$ and (1.10) hold for all integer $n$.

Note that $P_{n}$ and $S_{n}$ are two variants of the same sequence in [71]. In fact, the following are basically all variants of the same sequence in [71] which $P_{n}$ and $S_{n}$ belong: A000931, A078027, A096231, A124745, A133034, A134816, A164001, A182097, A228361 and probably A020720 (however, each one has its own special features and deserves its own entry). $E_{n}$ is the sequence A001608 in [71] and $A_{n}$ is the sequence A276276 in [71].

Next, we present the first few values of the Padovan, Perrin, Padovan-Perrin and modified Padovan numbers with positive and negative subscripts:

Table 2. The first few values of the special third-order numbers with positive and negative subscripts.

\begin{tabular}{ccccccccccccccc}
\hline$n$ & 0 & 1 & 2 & 3 & 4 & 5 & 6 & 7 & 8 & 9 & 10 & 11 & 12 & 13 \\
\hline$P_{n}$ & 1 & 1 & 1 & 2 & 2 & 3 & 4 & 5 & 7 & 9 & 12 & 16 & 21 & 28 \\
$P_{-n}$ & & 0 & 1 & 0 & 0 & 1 & -1 & 1 & 0 & -1 & 2 & -2 & 1 & 1 \\
$E_{n}$ & 3 & 0 & 2 & 3 & 2 & 5 & 5 & 7 & 10 & 12 & 17 & 22 & 29 & 39 \\
$E_{-n}$ & & -1 & 1 & 2 & -3 & 4 & -2 & -1 & 5 & -7 & 6 & -1 & -6 & 12 \\
$S_{n}$ & 0 & 0 & 1 & 0 & 1 & 1 & 1 & 2 & 2 & 3 & 4 & 5 & 7 & 9 \\
$S_{-n}$ & & 1 & -1 & 1 & 0 & -1 & 2 & -2 & 1 & 1 & -3 & 4 & -3 & 0 \\
$A_{n}$ & 3 & 1 & 3 & 4 & 4 & 7 & 8 & 11 & 15 & 19 & 26 & 34 & 45 & 60 \\
$A_{-n}$ & 0 & 1 & 2 & -2 & 3 & -1 & -1 & 4 & -5 & 4 & 0 & -5 & 9 \\
\hline
\end{tabular}


For all integers $n$, Padovan, Perrin, Padovan-Perrin and modified Padovan numbers (using initial conditions in (1.6)) can be expressed using Binet's formulas as

$$
\begin{aligned}
P_{n} & =\frac{\alpha^{n+4}}{(\alpha-\beta)(\alpha-\gamma)}+\frac{\beta^{n+4}}{(\beta-\alpha)(\beta-\gamma)}+\frac{\gamma^{n+4}}{(\gamma-\alpha)(\gamma-\beta)}, \\
E_{n} & =\alpha^{n}+\beta^{n}+\gamma^{n}, \\
S_{n} & =\frac{\alpha^{n}}{(\alpha-\beta)(\alpha-\gamma)}+\frac{\beta^{n}}{(\beta-\alpha)(\beta-\gamma)}+\frac{\gamma^{n}}{(\gamma-\alpha)(\gamma-\beta)}, \\
A_{n} & =\frac{(3 \alpha+1) \alpha^{n+1}}{(\alpha-\beta)(\alpha-\gamma)}+\frac{(3 \beta+1) \beta^{n+1}}{(\beta-\alpha)(\beta-\gamma)}+\frac{(3 \gamma+1) \gamma^{n+1}}{(\gamma-\alpha)(\gamma-\beta)},
\end{aligned}
$$

respectively. Note that $P_{n}, S_{n}$ and $A_{n}$ can be written as

$$
\begin{aligned}
P_{n} & =\frac{\alpha^{n+5}}{2 \alpha+3}+\frac{\beta^{n+5}}{2 \beta+3}+\frac{\gamma^{n+5}}{2 \gamma+3}, \\
S_{n} & =\frac{\alpha^{n+1}}{2 \alpha+3}+\frac{\beta^{n+1}}{2 \beta+3}+\frac{\gamma^{n+1}}{2 \gamma+3}, \\
A_{n} & =\frac{(3 \alpha+1) \alpha^{n+2}}{2 \alpha+3}+\frac{(3 \beta+1) \beta^{n+2}}{2 \beta+3}+\frac{(3 \gamma+1) \gamma^{n+2}}{2 \gamma+3} .
\end{aligned}
$$

\section{Generating Functions}

Next, we give the ordinary generating function $\sum_{n=0}^{\infty} V_{n} x^{n}$ of the sequence $V_{n}$.

Lemma 1. Suppose that $f_{V_{n}}(x)=\sum_{n=0}^{\infty} V_{n} x^{n}$ is the ordinary generating function of the generalized Padovan sequence $\left\{V_{n}\right\}_{n \geq 0}$. Then, $\sum_{n=0}^{\infty} V_{n} x^{n}$ is given by

$$
\sum_{n=0}^{\infty} V_{n} x^{n}=\frac{V_{0}+V_{1} x+\left(V_{2}-V_{0}\right) x^{2}}{1-x^{2}-x^{3}}
$$

Proof. Using the definition of generalized Padovan numbers, and substracting $x^{2} \sum_{n=0}^{\infty} V_{n} x^{n}$ and $x^{3} \sum_{n=0}^{\infty} V_{n} x^{n}$ from $\sum_{n=0}^{\infty} V_{n} x^{n}$ we obtain

$$
\begin{aligned}
\left(1-x^{2}-x^{3}\right) \sum_{n=0}^{\infty} V_{n} x^{n}= & \sum_{n=0}^{\infty} V_{n} x^{n}-x^{2} \sum_{n=0}^{\infty} V_{n} x^{n}-x^{3} \sum_{n=0}^{\infty} V_{n} x^{n} \\
= & \sum_{n=0}^{\infty} V_{n} x^{n}-\sum_{n=0}^{\infty} V_{n} x^{n+2}-\sum_{n=0}^{\infty} V_{n} x^{n+3} \\
= & \sum_{n=0}^{\infty} V_{n} x^{n}-\sum_{n=2}^{\infty} V_{n-2} x^{n}-\sum_{n=3}^{\infty} V_{n-3} x^{n} \\
= & \left(V_{0}+V_{1} x+V_{2} x^{2}\right)-V_{0} x^{2} \\
& +\sum_{n=3}^{\infty}\left(V_{n}-V_{n-2}-V_{n-3}\right) x^{n} \\
= & V_{0}+V_{1} x+V_{2} x^{2}-V_{0} x^{2} \\
= & V_{0}+V_{1} x+\left(V_{2}-V_{0}\right) x^{2} .
\end{aligned}
$$


Rearranging above equation, we obtain

$$
\sum_{n=0}^{\infty} V_{n} x^{n}=\frac{V_{0}+V_{1} x+\left(V_{2}-V_{0}\right) x^{2}}{1-x^{2}-x^{3}} .
$$

The previous lemma gives the following results as particular examples.

Corollary 2. Generated functions of Padovan, Perrin, Padovan-Perrin and modified Padovan numbers are

$$
\begin{aligned}
\sum_{n=0}^{\infty} P_{n} x^{n} & =\frac{1+x}{1-x^{2}-x^{3}}, \\
\sum_{n=0}^{\infty} E_{n} x^{n} & =\frac{3-x^{2}}{1-x^{2}-x^{3}}, \\
\sum_{n=0}^{\infty} S_{n} x^{n} & =\frac{x^{2}}{1-x^{2}-x^{3}}, \\
\sum_{n=0}^{\infty} A_{n} x^{n} & =\frac{3+x}{1-x^{2}-x^{3}},
\end{aligned}
$$

respectively.

\section{Obtaining Binet Formula From Generating Function}

We next find Binet formula of generalized Grahaml numbers $\left\{V_{n}\right\}$ by the use of generating function for $V_{n}$.

THEOREM 3. (Binet formula of generalized Padovan numbers)

$$
V_{n}=\frac{d_{1} \alpha^{n}}{(\alpha-\beta)(\alpha-\gamma)}+\frac{d_{2} \beta^{n}}{(\beta-\alpha)(\beta-\gamma)}+\frac{d_{3} \gamma^{n}}{(\gamma-\alpha)(\gamma-\beta)}
$$

where

$$
\begin{aligned}
& d_{1}=V_{0} \alpha^{2}+V_{1} \alpha+\left(V_{2}-V_{0}\right), \\
& d_{2}=V_{0} \beta^{2}+V_{1} \beta+\left(V_{2}-V_{0}\right), \\
& d_{3}=V_{0} \gamma^{2}+V_{1} \gamma+\left(V_{2}-V_{0}\right) .
\end{aligned}
$$

Proof. Let

$$
h(x)=1-x^{2}-x^{3} .
$$

Then for some $\alpha, \beta$ and $\gamma$ we write

$$
h(x)=(1-\alpha x)(1-\beta x)(1-\gamma x)
$$

i.e.,

$$
1-x^{2}-x^{3}=(1-\alpha x)(1-\beta x)(1-\gamma x)
$$


Hence $\frac{1}{\alpha}, \frac{1}{\beta}$, ve $\frac{1}{\gamma}$ are the roots of $h(x)$. This gives $\alpha, \beta$, and $\gamma$ as the roots of

$$
h\left(\frac{1}{x}\right)=1-\frac{1}{x^{2}}-\frac{1}{x^{3}}=0 .
$$

This implies $x^{3}-x-1=0$. Now, by (2.1) and (3.2), it follows that

$$
\sum_{n=0}^{\infty} V_{n} x^{n}=\frac{V_{0}+V_{1} x+\left(V_{2}-V_{0}\right) x^{2}}{(1-\alpha x)(1-\beta x)(1-\gamma x)}
$$

Then we write

$$
\frac{V_{0}+V_{1} x+\left(V_{2}-V_{0}\right) x^{2}}{(1-\alpha x)(1-\beta x)(1-\gamma x)}=\frac{A_{1}}{(1-\alpha x)}+\frac{A_{2}}{(1-\beta x)}+\frac{A_{3}}{(1-\gamma x)}
$$

So

$$
V_{0}+V_{1} x+\left(V_{2}-V_{0}\right) x^{2}=A_{1}(1-\beta x)(1-\gamma x)+A_{2}(1-\alpha x)(1-\gamma x)+A_{3}(1-\alpha x)(1-\beta x) .
$$

If we consider $x=\frac{1}{\alpha}$, we get $V_{0}+V_{1} \frac{1}{\alpha}+\left(V_{2}-V_{0}\right) \frac{1}{\alpha^{2}}=A_{1}\left(1-\frac{\beta}{\alpha}\right)\left(1-\frac{\gamma}{\alpha}\right)$. This gives

$$
A_{1}=\frac{\alpha^{2}\left(V_{0}+V_{1} \frac{1}{\alpha}+\left(V_{2}-V_{0}\right) \frac{1}{\alpha^{2}}\right)}{(\alpha-\beta)(\alpha-\gamma)}=\frac{V_{0} \alpha^{2}+V_{1} \alpha+\left(V_{2}-V_{0}\right)}{(\alpha-\beta)(\alpha-\gamma)}
$$

Similarly, we obtain

$$
A_{2}=\frac{V_{0} \beta^{2}+V_{1} \beta+\left(V_{2}-V_{0}\right)}{(\beta-\alpha)(\beta-\gamma)}, A_{3}=\frac{V_{0} \gamma^{2}+V_{1} \gamma+\left(V_{2}-V_{0}\right)}{(\gamma-\alpha)(\gamma-\beta)}
$$

Thus (3.3) can be written as

$$
\sum_{n=0}^{\infty} V_{n} x^{n}=A_{1}(1-\alpha x)^{-1}+A_{2}(1-\beta x)^{-1}+A_{3}(1-\gamma x)^{-1} .
$$

This gives

$$
\sum_{n=0}^{\infty} V_{n} x^{n}=A_{1} \sum_{n=0}^{\infty} \alpha^{n} x^{n}+A_{2} \sum_{n=0}^{\infty} \beta^{n} x^{n}+A_{3} \sum_{n=0}^{\infty} \gamma^{n} x^{n}=\sum_{n=0}^{\infty}\left(A_{1} \alpha^{n}+A_{2} \beta^{n}+A_{3} \gamma^{n}\right) x^{n}
$$

Therefore, comparing coefficients on both sides of the above equality, we obtain

$$
V_{n}=A_{1} \alpha^{n}+A_{2} \beta^{n}+A_{3} \gamma^{n}
$$

where

$$
\begin{aligned}
& A_{1}=\frac{V_{0} \alpha^{2}+V_{1} \alpha+\left(V_{2}-V_{0}\right)}{(\alpha-\beta)(\alpha-\gamma)}, \\
& A_{2}=\frac{V_{0} \beta^{2}+V_{1} \beta+\left(V_{2}-V_{0}\right)}{(\beta-\alpha)(\beta-\gamma)} \\
& A_{3}=\frac{V_{0} \gamma^{2}+V_{1} \gamma+\left(V_{2}-V_{0}\right)}{(\gamma-\alpha)(\gamma-\beta)} .
\end{aligned}
$$

and then we get (3.1). 
Note that from (1.6) and (3.1) we have

$$
\begin{aligned}
V_{2}-(\beta+\gamma) V_{1}+\beta \gamma V_{0} & =V_{0} \alpha^{2}+V_{1} \alpha+\left(V_{2}-V_{0}\right) \\
V_{2}-(\alpha+\gamma) V_{1}+\alpha \gamma V_{0} & =V_{0} \beta^{2}+V_{1} \beta+\left(V_{2}-V_{0}\right) \\
V_{2}-(\alpha+\beta) V_{1}+\alpha \beta V_{0} & =V_{0} \gamma^{2}+V_{1} \gamma+\left(V_{2}-V_{0}\right) .
\end{aligned}
$$

Next, using Theorem 3, we present the Binet formulas of Padovan, Perrin, Padovan-Perrin and modified Padovan sequences.

Corollary 4. Binet formulas of Padovan, Perrin, Padovan-Perrin and modified Padovan sequences are

$$
\begin{aligned}
& P_{n}=\frac{(\alpha+1) \alpha^{n+1}}{(\alpha-\beta)(\alpha-\gamma)}+\frac{(\beta+1) \beta^{n+1}}{(\beta-\alpha)(\beta-\gamma)}+\frac{(\gamma+1) \gamma^{n+1}}{(\gamma-\alpha)(\gamma-\beta)}, \\
& E_{n}=\alpha^{n}+\beta^{n}+\gamma^{n}, \\
& S_{n}=\frac{\alpha^{n}}{(\alpha-\beta)(\alpha-\gamma)}+\frac{\beta^{n}}{(\beta-\alpha)(\beta-\gamma)}+\frac{\gamma^{n}}{(\gamma-\alpha)(\gamma-\beta)}, \\
& A_{n}=\frac{(3 \alpha+1) \alpha^{n+1}}{(\alpha-\beta)(\alpha-\gamma)}+\frac{(3 \beta+1) \beta^{n+1}}{(\beta-\alpha)(\beta-\gamma)}+\frac{(3 \gamma+1) \gamma^{n+1}}{(\gamma-\alpha)(\gamma-\beta)},
\end{aligned}
$$

respectively.

We can find Binet formulas by using matrix method with a similar technique which is given in [47]. Take $k=i=3$ in Corollary 3.1 in [47]. Let

$$
\begin{aligned}
\Lambda & =\left(\begin{array}{lll}
\alpha^{2} & \alpha & 1 \\
\beta^{2} & \beta & 1 \\
\gamma^{2} & \gamma & 1
\end{array}\right), \Lambda_{1}=\left(\begin{array}{ccc}
\alpha^{n-1} & \alpha & 1 \\
\beta^{n-1} & \beta & 1 \\
\gamma^{n-1} & \gamma & 1
\end{array}\right), \\
\Lambda_{2} & =\left(\begin{array}{llll}
\alpha^{2} & \alpha^{n-1} & 1 \\
\beta^{2} & \beta^{n-1} & 1 \\
\gamma^{2} & \gamma^{n-1} & 1
\end{array}\right), \Lambda_{3}=\left(\begin{array}{ccc}
\alpha^{2} & \alpha & \alpha^{n-1} \\
\beta^{2} & \beta & \beta^{n-1} \\
\gamma^{2} & \gamma & \gamma^{n-1}
\end{array}\right) .
\end{aligned}
$$

Then the Binet formula for Padovan numbers is

$$
\begin{aligned}
P_{n} & =\frac{1}{\operatorname{det}(\Lambda)} \sum_{j=1}^{3} P_{4-j} \operatorname{det}\left(\Lambda_{j}\right)=\frac{1}{\Lambda}\left(P_{3} \operatorname{det}\left(\Lambda_{1}\right)+P_{2} \operatorname{det}\left(\Lambda_{2}\right)+P_{1} \operatorname{det}\left(\Lambda_{3}\right)\right) \\
& =\frac{1}{\operatorname{det}(\Lambda)}\left(2 \operatorname{det}\left(\Lambda_{1}\right)+\operatorname{det}\left(\Lambda_{2}\right)+\operatorname{det}\left(\Lambda_{3}\right)\right) \\
& =\left(2\left|\begin{array}{lll}
\alpha^{n-1} & \alpha & 1 \\
\beta^{n-1} & \beta & 1 \\
\gamma^{n-1} & \gamma & 1
\end{array}\right|+\left|\begin{array}{lll}
\alpha^{2} & \alpha^{n-1} & 1 \\
\beta^{2} & \beta^{n-1} & 1 \\
\gamma^{2} & \gamma^{n-1} & 1
\end{array}\right|+\left|\begin{array}{ccc}
\alpha^{2} & \alpha & \alpha^{n-1} \\
\beta^{2} & \beta & \beta^{n-1} \\
\gamma^{2} & \gamma & \gamma^{n-1}
\end{array}\right|\right) /\left|\begin{array}{ccc}
\alpha^{2} & \alpha & 1 \\
\beta^{2} & \beta & 1 \\
\gamma^{2} & \gamma & 1
\end{array}\right| .
\end{aligned}
$$


Similarly, we obtain the Binet formula for Perrin, Padovan-Perrin and modified Padovan as

$$
\begin{aligned}
E_{n}= & \frac{1}{\Lambda}\left(E_{3} \operatorname{det}\left(\Lambda_{1}\right)+E_{2} \operatorname{det}\left(\Lambda_{2}\right)+E_{1} \operatorname{det}\left(\Lambda_{3}\right)\right) \\
= & \left(3\left|\begin{array}{lll}
\alpha^{n-1} & \alpha & 1 \\
\beta^{n-1} & \beta & 1 \\
\gamma^{n-1} & \gamma & 1
\end{array}\right|+2\left|\begin{array}{ccc}
\alpha^{2} & \alpha^{n-1} & 1 \\
\beta^{2} & \beta^{n-1} & 1 \\
\gamma^{2} & \gamma^{n-1} & 1
\end{array}\right|\right) /\left|\begin{array}{lll}
\alpha^{2} & \alpha & 1 \\
\beta^{2} & \beta & 1 \\
\gamma^{2} & \gamma & 1
\end{array}\right|
\end{aligned}
$$

and

$$
\begin{aligned}
S_{n} & =\frac{1}{\Lambda}\left(S_{3} \operatorname{det}\left(\Lambda_{1}\right)+S_{2} \operatorname{det}\left(\Lambda_{2}\right)+S_{1} \operatorname{det}\left(\Lambda_{3}\right)\right) \\
& =\left|\begin{array}{lll}
\alpha^{2} & \alpha^{n-1} & 1 \\
\beta^{2} & \beta^{n-1} & 1 \\
\gamma^{2} & \gamma^{n-1} & 1
\end{array}\right| /\left|\begin{array}{ccc}
\alpha^{2} & \alpha & 1 \\
\beta^{2} & \beta & 1 \\
\gamma^{2} & \gamma & 1
\end{array}\right| .
\end{aligned}
$$

and

$$
\begin{aligned}
A_{n} & =\frac{1}{\Lambda}\left(A_{3} \operatorname{det}\left(\Lambda_{1}\right)+A_{2} \operatorname{det}\left(\Lambda_{2}\right)+A_{1} \operatorname{det}\left(\Lambda_{3}\right)\right) \\
& =\left(4\left|\begin{array}{lll}
\alpha^{n-1} & \alpha & 1 \\
\beta^{n-1} & \beta & 1 \\
\gamma^{n-1} & \gamma & 1
\end{array}\right|+3\left|\begin{array}{ccc}
\alpha^{2} & \alpha^{n-1} & 1 \\
\beta^{2} & \beta^{n-1} & 1 \\
\gamma^{2} & \gamma^{n-1} & 1
\end{array}\right|+\left|\begin{array}{ccc}
\alpha^{2} & \alpha & \alpha^{n-1} \\
\beta^{2} & \beta & \beta^{n-1} \\
\gamma^{2} & \gamma & \gamma^{n-1}
\end{array}\right|\right) /\left|\begin{array}{ccc}
\alpha^{2} & \alpha & 1 \\
\beta^{2} & \beta & 1 \\
\gamma^{2} & \gamma & 1
\end{array}\right|
\end{aligned}
$$

respectively.

\section{Simson Formulas}

There is a well-known Simson Identity (formula) for Fibonacci sequence $\left\{F_{n}\right\}$, namely,

$$
F_{n+1} F_{n-1}-F_{n}^{2}=(-1)^{n}
$$

which was derived first by R. Simson in 1753 and it is now called as Cassini Identity (formula) as well. This can be written in the form

$$
\left|\begin{array}{cc}
F_{n+1} & F_{n} \\
F_{n} & F_{n-1}
\end{array}\right|=(-1)^{n} .
$$

The following theorem gives generalization of this result to the generalized Padovan sequence $\left\{V_{n}\right\}_{n \geq 0}$.

TheOREM 5 (Simson Formula of Generalized Padovan Numbers). For all integers n, we have

$$
\left|\begin{array}{ccc}
V_{n+2} & V_{n+1} & V_{n} \\
V_{n+1} & V_{n} & V_{n-1} \\
V_{n} & V_{n-1} & V_{n-2}
\end{array}\right|=\left|\begin{array}{ccc}
V_{2} & V_{1} & V_{0} \\
V_{1} & V_{0} & V_{-1} \\
V_{0} & V_{-1} & V_{-2}
\end{array}\right| .
$$

Proof. (4.1) is given in Soykan [76].

The previous theorem gives the following results as particular examples. 
Corollary 6. For all integers n, Simson formula of Padovan, Perrin, Padovan-Perrin and modified Padovan numbers are given as

and

$$
\left|\begin{array}{ccc}
P_{n+2} & P_{n+1} & P_{n} \\
P_{n+1} & P_{n} & P_{n-1} \\
P_{n} & P_{n-1} & P_{n-2}
\end{array}\right|=-1
$$

$\left|\begin{array}{ccc}E_{n+2} & E_{n+1} & E_{n} \\ E_{n+1} & E_{n} & E_{n-1} \\ E_{n} & E_{n-1} & E_{n-2}\end{array}\right|=-23$

and

respectively.

$$
\left|\begin{array}{ccc}
S_{n+2} & S_{n+1} & S_{n} \\
S_{n+1} & S_{n} & S_{n-1} \\
S_{n} & S_{n-1} & S_{n-2}
\end{array}\right|=-1
$$

and

\section{Some Identities}

In this section, we obtain some identities of Padovan, Perrin, Padovan-Perrin and modified Padovan numbers. First, we can give a few basic relations between $\left\{P_{n}\right\}$ and $\left\{E_{n}\right\}$.

LEMma 7. The following equalities are true:

$$
\begin{aligned}
& E_{n}=-2 P_{n+4}+4 P_{n+3}-P_{n+2}, \\
& E_{n}=4 P_{n+3}-3 P_{n+2}-2 P_{n+1}, \\
& E_{n}=-3 P_{n+2}+2 P_{n+1}+4 P_{n}, \\
& E_{n}=2 P_{n+1}+P_{n}-3 P_{n-1}, \\
& E_{n}=P_{n}-P_{n-1}+2 P_{n-2},
\end{aligned}
$$

and

$$
\begin{aligned}
& 23 P_{n}=-2 E_{n+4}+3 E_{n+3}+9 E_{n+2}, \\
& 23 P_{n}=3 E_{n+3}+7 E_{n+2}-2 E_{n+1}, \\
& 23 P_{n}=7 E_{n+2}+E_{n+1}+3 E_{n}, \\
& 23 P_{n}=E_{n+1}+10 E_{n}+7 E_{n-1}, \\
& 23 P_{n}=10 E_{n}+8 E_{n-1}+E_{n-2} .
\end{aligned}
$$


Proof. Note that all the identities hold for all integers $n$. We prove (5.1). To show (5.1), writing

$$
E_{n}=a \times P_{n+4}+b \times P_{n+3}+c \times P_{n+2}
$$

and solving the system of equations

$$
\begin{aligned}
& E_{0}=a \times P_{4}+b \times P_{3}+c \times P_{2} \\
& E_{1}=a \times P_{5}+b \times P_{4}+c \times P_{3} \\
& E_{2}=a \times P_{6}+b \times P_{5}+c \times P_{4}
\end{aligned}
$$

we find that $a=-2, b=4, c=-1$. The other equalities can be proved similarly.

Note that all the identities in the above Lemma can be proved by induction as well.

Next, we present a few basic relations between $\left\{P_{n}\right\}$ and $\left\{S_{n}\right\}$.

Lemma 8. The following equalities are true:

$$
\begin{aligned}
S_{n} & =2 P_{n+4}-P_{n+3}-2 P_{n+2}, \\
S_{n} & =-P_{n+3}+2 P_{n+1}, \\
S_{n} & =P_{n+1}-P_{n}, \\
S_{n} & =-P_{n}+P_{n-1}+P_{n-2},
\end{aligned}
$$

and

$$
\begin{aligned}
& P_{n}=S_{n+4}, \\
& P_{n}=S_{n+2}+S_{n+1}, \\
& P_{n}=S_{n+1}+S_{n}+S_{n-1}, \\
& P_{n}=S_{n}+2 S_{n-1}+S_{n-2} .
\end{aligned}
$$

Now, we give a few basic relations between $\left\{P_{n}\right\}$ and $\left\{A_{n}\right\}$.

Lemma 9. The following equalities are true:

$$
\begin{aligned}
& A_{n}=-P_{n+4}+3 P_{n+3}-P_{n+2}, \\
& A_{n}=3 P_{n+3}-2 P_{n+2}-P_{n+1}, \\
& A_{n}=-2 P_{n+2}+2 P_{n+1}+3 P_{n}, \\
& A_{n}=2 P_{n+1}+P_{n}-2 P_{n-1}, \\
& A_{n}=P_{n}+2 P_{n-2},
\end{aligned}
$$


and

$$
\begin{aligned}
& 19 P_{n}=-3 A_{n+4}+A_{n+3}+9 A_{n+2}, \\
& 19 P_{n}=A_{n+3}+6 A_{n+2}-3 A_{n+1}, \\
& 19 P_{n}=6 A_{n+2}-2 A_{n+1}+A_{n}, \\
& 19 P_{n}=-2 A_{n+1}+7 A_{n}+6 \times A_{n-1}, \\
& 19 P_{n}=7 A_{n}+4 A_{n-1}-2 A_{n-2} .
\end{aligned}
$$

Next, we present a few basic relations between $\left\{E_{n}\right\}$ and $\left\{S_{n}\right\}$.

LEMma 10. The following equalities are true

$$
\begin{aligned}
& 23 S_{n}=5 E_{n+4}+4 E_{n+3}-11 E_{n+2}, \\
& 23 S_{n}=4 E_{n+3}-6 E_{n+2}+5 E_{n+1} \\
& 23 S_{n}=-6 E_{n+2}+9 E_{n+1}+4 E_{n} \\
& 23 S_{n}=9 E_{n+1}-2 E_{n}-6 E_{n-1}, \\
& 23 S_{n}=-2 E_{n}+3 E_{n-1}+9 E_{n-2} .
\end{aligned}
$$

and

$$
\begin{aligned}
& E_{n}=S_{n+4}-S_{n+3}+2 S_{n+2} \\
& E_{n}=-S_{n+3}+3 S_{n+2}+S_{n+1} \\
& E_{n}=3 S_{n+2}-S_{n} \\
& E_{n}=2 S_{n}+3 S_{n-1} .
\end{aligned}
$$

Next, we give a few basic relations between $\left\{A_{n}\right\}$ and $\left\{E_{n}\right\}$.

LEMMA 11. The following equalities are true

$$
\begin{aligned}
19 E_{n} & =-28 A_{n+4}+22 A_{n+3}+27 A_{n+2}, \\
19 E_{n} & =22 A_{n+3}-A_{n+2}-28 A_{n+1} \\
19 E_{n} & =-A_{n+2}-6 A_{n+1}+22 A_{n} \\
19 E_{n} & =-6 A_{n+1}+21 A_{n}-A_{n-1} \\
19 E_{n} & =21 A_{n}-7 A_{n-1}-6 A_{n-2}
\end{aligned}
$$


and

$$
\begin{aligned}
& 23 A_{n}=-14 E_{n+4}+21 E_{n+3}+17 E_{n+2}, \\
& 23 A_{n}=21 E_{n+3}+3 E_{n+2}-14 E_{n+1}, \\
& 23 A_{n}=3 E_{n+2}+7 E_{n+1}+21 E_{n} \\
& 23 A_{n}=7 E_{n+1}+24 E_{n}+3 E_{n-1} \\
& 23 A_{n}=24 E_{n}+10 E_{n-1}+7 E_{n-2} .
\end{aligned}
$$

Now, we present a few basic relations between $\left\{S_{n}\right\}$ and $\left\{A_{n}\right\}$.

LEMma 12. The following equalities are true

$$
\begin{aligned}
& A_{n}=S_{n+4}+2 S_{n+2}, \\
& A_{n}=3 S_{n+2}+S_{n+1}, \\
& A_{n}=3 S_{n+2}+S_{n+1}, \\
& A_{n}=S_{n+1}+3 S_{n}+3 S_{n-1}, \\
& A_{n}=3 S_{n}+4 S_{n-1}+S_{n-2},
\end{aligned}
$$

and

$$
\begin{aligned}
19 S_{n} & =4 A_{n+4}+5 A_{n+3}-12 A_{n+2}, \\
19 S_{n} & =5 A_{n+3}-8 A_{n+2}+4 A_{n+1}, \\
19 S_{n} & =-8 A_{n+2}+9 A_{n+1}+5 A_{n}, \\
19 S_{n} & =9 A_{n+1}-3 A_{n}-8 A_{n-1}, \\
19 S_{n} & =-3 A_{n}+A_{n-1}+9 A_{n-2} .
\end{aligned}
$$

We now present a few special identities for the modified Padovan sequence $\left\{A_{n}\right\}$.

THEOREM 13. (Catalan's identity) For all integers $n$ and $m$, the following identity holds

$$
\begin{aligned}
A_{n+m} A_{n-m}-A_{n}^{2}= & \left(P_{n+m}+2 P_{n+m-2}\right)\left(P_{n-m}+2 P_{n-m-2}\right)-\left(P_{n}+2 P_{n-2}\right)^{2} \\
= & -P_{n}^{2}-4 P_{n} P_{n-2}-4 P_{n-2}^{2}+P_{m+n} P_{n-m}+2 P_{m+n} P_{n-m-2} \\
& +2 P_{m+n-2} P_{n-m}+4 P_{m+n-2} P_{n-m-2} .
\end{aligned}
$$

Proof. We use the identity

$$
A_{n}=P_{n}+2 P_{n-2}
$$

Note that for $m=1$ in Catalan's identity, we get the Cassini identity for the modified Padovan sequnce 
Corollary 14. (Cassini's identity) For all integers numbers $n$ and $m$, the following identity holds

$$
\begin{aligned}
A_{n+1} A_{n-1}-A_{n}^{2} & =\left(P_{n+1}+2 P_{n+1-2}\right)\left(P_{n-1}+2 P_{n-1-2}\right)-\left(P_{n}+2 P_{n-2}\right)^{2} \\
& =P_{n-1} P_{n+1}-P_{n}^{2}-4 P_{n} P_{n-2}+4 P_{n-1} P_{n-3}+2 P_{n+1} P_{n-3}+2 P_{n-1}^{2}-4 P_{n-2}^{2}
\end{aligned}
$$

The d'Ocagne's, Gelin-Cesàro's and Melham' identities can also be obtained by using $A_{n}=P_{n}+$ $2 P_{n-2}$. The next theorem presents d'Ocagne's, Gelin-Cesàro's and Melham' identities of modified Padovan sequence $\left\{A_{n}\right\}$.

THEOREM 15. Let $n$ and $m$ be any integers. Then the following identities are true:

(a): (d'Ocagne's identity)

$$
A_{m+1} A_{n}-A_{m} A_{n+1}=\left(P_{m+1}+2 P_{m+1-2}\right)\left(P_{n}+2 P_{n-2}\right)-\left(P_{m}+2 P_{m-2}\right)\left(P_{n+1}+2 P_{n+1-2}\right) .
$$

(b): (Gelin-Cesàro's identity)

$$
A_{n+2} A_{n+1} A_{n-1} A_{n-2}-A_{n}^{4}=\left(P_{n+2}+2 P_{n+2-2}\right)\left(P_{n+1}+2 P_{n+1-2}\right)\left(P_{n-1}+2 P_{n-1-2}\right)\left(P_{n-2}+2 P_{n-2-2}\right)-\left(P_{n}+2 P_{n-2}\right)^{4} .
$$

(c): (Melham's identity)

$$
\begin{aligned}
A_{n+1} A_{n+2} A_{n+6}-A_{n+3}^{3} & =\left(P_{n+1}+2 P_{n+1-2}\right)\left(P_{n+2}+2 P_{n+2-2}\right)\left(P_{n+6}+2 P_{n+6-2}\right)-\left(P_{n}+2 P_{n-2}\right)^{3} \\
& =\left(P_{n+1}+2 P_{n-1}\right)\left(P_{n+2}+2 P_{n}\right)\left(P_{n+6}+2 P_{n+4}\right)-\left(P_{n}+2 P_{n-2}\right)^{3}
\end{aligned}
$$

Proof. Use the identity $A_{n}=P_{n}+2 P_{n-2}$.

\section{Linear Sums}

The following proposition presents some formulas of generalized Padovan numbers with positive subscripts.

Proposition 16. If $r=0, s=1, t=1$ then for $n \geq 0$ we have the following formulas:

(a): $\sum_{k=0}^{n} V_{k}=V_{n+3}+V_{n+2}-V_{2}-V_{1}$.

(b): $\sum_{k=0}^{n} V_{2 k}=V_{2 n+1}+V_{2 n}-V_{1}$.

(c): $\sum_{k=0}^{n} V_{2 k+1}=V_{2 n+2}+V_{2 n+1}-V_{2}$.

Proof. Take $r=0, s=1, t=1$ in Theorem 2.1 in [75].

As special cases of above proposition, we have the following four corollaries. First one presents some summing formulas of Padovan numbers (take $V_{n}=P_{n}$ with $P_{0}=1, P_{1}=1, P_{2}=1$ ).

COROLlary 17. For $n \geq 0$ we have the following formulas:

(a): $\sum_{k=0}^{n} P_{k}=P_{n+3}+P_{n+2}-2$.

(b): $\sum_{k=0}^{n} P_{2 k}=P_{2 n+1}+P_{2 n}-1$.

(c): $\sum_{k=0}^{n} P_{2 k+1}=P_{2 n+2}+P_{2 n+1}-1$. 
Second one presents some summing formulas of Perrin (Padovan-Lucas) numbers (take $V_{n}=E_{n}$ with $\left.E_{0}=3, E_{1}=0, E_{2}=2\right)$.

COROLlary 18. For $n \geq 0$ we have the following formulas:
(a): $\sum_{k=0}^{n} E_{k}=E_{n+3}+E_{n+2}-2$.
(b): $\sum_{k=0}^{n} E_{2 k}=E_{2 n+1}+E_{2 n}$.
(c): $\sum_{k=0}^{n} E_{2 k+1}=E_{2 n+2}+E_{2 n+1}-2$.

Third one presents some summing formulas of Padovan-Perrin numbers (take $V_{n}=S_{n}$ with $S_{0}=0, S_{1}=$ $\left.0, S_{2}=1\right)$.

COROLlary 19. For $n \geq 0$ we have the following formulas:
(a): $\sum_{k=0}^{n} S_{k}=S_{n+3}+S_{n+2}-1$.
(b): $\sum_{k=0}^{n} S_{2 k}=S_{2 n+1}+S_{2 n}$.
(c): $\sum_{k=0}^{n} S_{2 k+1}=S_{2 n+2}+S_{2 n+1}-1$.

Fourth one presents some summing formulas of modified Padovan numbers (take $V_{n}=A_{n}$ with $A_{0}=$ $\left.3, A_{1}=1, A_{2}=3\right)$.

COROLlary 20. For $n \geq 0$ we have the following formulas:
(a): $\sum_{k=0}^{n} A_{k}=A_{n+3}+A_{n+2}-4$.
(b): $\sum_{k=0}^{n} A_{2 k}=A_{2 n+1}+A_{2 n}-1$.
(c): $\sum_{k=0}^{n} A_{2 k+1}=A_{2 n+2}+A_{2 n+1}-3$.

The following proposition presents some formulas of generalized Padovan numbers with negative subscripts.

Proposition 21. If $r=0, s=1, t=1$ then for $n \geq 1$ we have the following formulas:

(a): $\sum_{k=1}^{n} V_{-k}=-2 V_{-n-1}-2 V_{-n-2}-V_{-n-3}+V_{2}+V_{1}$.

(b): $\sum_{k=1}^{n} V_{-2 k}=-V_{-2 n+1}+V_{1}$.

(c): $\sum_{k=1}^{n} V_{-2 k+1}=-V_{-2 n}-V_{-2 n-1}+V_{2}$.

Proof. Take $r=0, s=1, t=1$ in Theorem 3.1 in [75].

From the above proposition, we have the following corollary which gives sum formulas of Padovan numbers (take $V_{n}=P_{n}$ with $P_{0}=1, P_{1}=1, P_{2}=1$ ).

Corollary 22. For $n \geq 1$, Padovan numbers have the following properties.
(a): $\sum_{k=1}^{n} P_{-k}=-2 P_{-n-1}-2 P_{-n-2}-P_{-n-3}+2$.
(b): $\sum_{k=1}^{n} P_{-2 k}=-P_{-2 n+1}+1$.
(c): $\sum_{k=1}^{n} P_{-2 k+1}=-P_{-2 n}-P_{-2 n-1}+1$. 
Taking $V_{n}=E_{n}$ with $E_{0}=3, E_{1}=0, E_{2}=2$ in the last proposition, we have the following corollary which presents sum formulas of Padovan-Lucas numbers.

Corollary 23. For $n \geq 1$, Perrin (Padovan-Lucas) numbers have the following properties.

(a): $\sum_{k=1}^{n} E_{-k}=-2 E_{-n-1}-2 E_{-n-2}-E_{-n-3}+2$.

(b): $\sum_{k=1}^{n} E_{-2 k}=-E_{-2 n+1}$.

(c): $\sum_{k=1}^{n} E_{-2 k+1}=-E_{-2 n}-E_{-2 n-1}+2$.

From the above proposition, we have the following corollary which gives sum formulas of Padovan-Perrin numbers (take $V_{n}=S_{n}$ with $S_{0}=0, S_{1}=0, S_{2}=1$ ).

Corollary 24. For $n \geq 1$, Padovan-Perrin numbers have the following properties.

(a): $\sum_{k=1}^{n} S_{-k}=-2 S_{-n-1}-2 S_{-n-2}-S_{-n-3}+1$.

(b): $\sum_{k=1}^{n} S_{-2 k}=-S_{-2 n+1}$.

(c): $\sum_{k=1}^{n} S_{-2 k+1}=-S_{-2 n}-S_{-2 n-1}+1$.

From the above proposition, we have the following corollary which gives sum formulas of modified Padovan numbers (take $V_{n}=A_{n}$ with $A_{0}=3, A_{1}=1, A_{2}=3$ ).

COROllary 25. For $n \geq 1$, modified Padovan numbers have the following properties.

(a): $\sum_{k=1}^{n} A_{-k}=-2 A_{-n-1}-2 A_{-n-2}-A_{-n-3}+4$.

(b): $\sum_{k=1}^{n} A_{-2 k}=-A_{-2 n+1}+1$.

(c): $\sum_{k=1}^{n} A_{-2 k+1}=-A_{-2 n}-A_{-2 n-1}+3$.

\section{Matrices related with Generalized Padovan numbers}

Matrix formulation of $W_{n}$ can be given as

$$
\left(\begin{array}{c}
W_{n+2} \\
W_{n+1} \\
W_{n}
\end{array}\right)=\left(\begin{array}{lll}
r & s & t \\
1 & 0 & 0 \\
0 & 1 & 0
\end{array}\right)^{n}\left(\begin{array}{l}
W_{2} \\
W_{1} \\
W_{0}
\end{array}\right) .
$$

For matrix formulation (7.1), see [41]. In fact, Kalman give the formula in the following form

$$
\left(\begin{array}{c}
W_{n} \\
W_{n+1} \\
W_{n+2}
\end{array}\right)=\left(\begin{array}{ccc}
0 & 1 & 0 \\
0 & 0 & 1 \\
r & s & t
\end{array}\right)^{n}\left(\begin{array}{c}
W_{0} \\
W_{1} \\
W_{2}
\end{array}\right) .
$$

We define the square matrix $A$ of order 3 as:

$$
A=\left(\begin{array}{lll}
0 & 1 & 1 \\
1 & 0 & 0 \\
0 & 1 & 0
\end{array}\right)
$$


such that $\operatorname{det} A=1$. From (1.5) we have

$$
\left(\begin{array}{c}
V_{n+2} \\
V_{n+1} \\
V_{n}
\end{array}\right)=\left(\begin{array}{lll}
0 & 1 & 1 \\
1 & 0 & 0 \\
0 & 1 & 0
\end{array}\right)\left(\begin{array}{c}
V_{n+1} \\
V_{n} \\
V_{n-1}
\end{array}\right)
$$

and from (7.1) (or using (7.2) and induction) we have

$$
\left(\begin{array}{c}
V_{n+2} \\
V_{n+1} \\
V_{n}
\end{array}\right)=\left(\begin{array}{lll}
0 & 1 & 1 \\
1 & 0 & 0 \\
0 & 1 & 0
\end{array}\right)^{n}\left(\begin{array}{c}
V_{2} \\
V_{1} \\
V_{0}
\end{array}\right) .
$$

If we take $V=P$ in $(7.2)$ we have

$$
\left(\begin{array}{c}
P_{n+2} \\
P_{n+1} \\
P_{n}
\end{array}\right)=\left(\begin{array}{lll}
0 & 1 & 1 \\
1 & 0 & 0 \\
0 & 1 & 0
\end{array}\right)\left(\begin{array}{c}
P_{n+1} \\
P_{n} \\
P_{n-1}
\end{array}\right) .
$$

We also define

$$
B_{n}=\left(\begin{array}{ccc}
P_{n-2} & P_{n-1} & P_{n-3} \\
P_{n-3} & P_{n-2} & P_{n-4} \\
P_{n-4} & P_{n-3} & P_{n-5}
\end{array}\right)
$$

and

$$
C_{n}=\left(\begin{array}{ccc}
V_{n-2} & V_{n-1} & V_{n-3} \\
V_{n-3} & V_{n-2} & V_{n-4} \\
V_{n-4} & V_{n-3} & V_{n-5}
\end{array}\right)
$$

Theorem 26. For all integer $m, n \geq 0$, we have
(a): $B_{n}=A^{n}$
(b): $C_{1} A^{n}=A^{n} C_{1}$
(c): $C_{n+m}=C_{n} B_{m}=B_{m} C_{n}$.

\section{Proof.}

(a): By expanding the vectors on the both sides of (7.3) to 3-colums and multiplying the obtained on the right-hand side by $A$, we get

$$
B_{n}=A B_{n-1}
$$

By induction argument, from the last equation, we obtain

$$
B_{n}=A^{n-1} B_{1}
$$

But $B_{1}=A$. It follows that $B_{n}=A^{n}$.

(b): Using (a) and definition of $C_{1}$, (b) follows. 
(c): We have

$$
\begin{aligned}
A C_{n-1} & =\left(\begin{array}{lll}
0 & 1 & 1 \\
1 & 0 & 0 \\
0 & 1 & 0
\end{array}\right)\left(\begin{array}{ccc}
V_{n-3} & V_{n-2} & V_{n-4} \\
V_{n-4} & V_{n-3} & V_{n-5} \\
V_{n-5} & V_{n-4} & V_{n-6}
\end{array}\right) \\
& =\left(\begin{array}{ccc}
V_{n-4}+V_{n-5} & V_{n-3}+V_{n-4} & V_{n-5}+V_{n-6} \\
V_{n-3} & V_{n-2} & V_{n-4} \\
V_{n-4} & V_{n-3} & V_{n-5}
\end{array}\right)=\left(\begin{array}{ccc}
V_{n-2} & V_{n-1} & V_{n-3} \\
V_{n-3} & V_{n-2} & V_{n-4} \\
V_{n-4} & V_{n-3} & V_{n-5}
\end{array}\right)=C_{n} .
\end{aligned}
$$

i.e. $C_{n}=A C_{n-1}$. From the last equation, using induction we obtain $C_{n}=A^{n-1} C_{1}$. Now

$$
C_{n+m}=A^{n+m-1} C_{1}=A^{n-1} A^{m} C_{1}=A^{n-1} C_{1} A^{m}=C_{n} B_{m}
$$

and similarly

$$
C_{n+m}=B_{m} C_{n}
$$

Some properties of matrix $A^{n}$ can be given as

$$
A^{n}=A^{n-2}+A^{n-3}
$$

and

$$
A^{n+m}=A^{n} A^{m}=A^{m} A^{n}
$$

and

$$
\operatorname{det}\left(A^{n}\right)=1
$$

for all integer $m$ and $n$.

Theorem 27. For $m, n \geq 0$ we have

$$
V_{n+m}=V_{n-2} P_{m}+V_{n-3} P_{m+1}+V_{n-4} P_{m-1} .
$$

Proof. From the equation $C_{n+m}=C_{n} B_{m}=B_{m} C_{n}$ we see that an element of $C_{n+m}$ is the product of row $C_{n}$ and a column $B_{m}$. From the last equation we say that an element of $C_{n+m}$ is the product of a row $C_{n}$ and column $B_{m}$. We just compare the linear combination of the 2 nd row and 1st column entries of the matrices $C_{n+m}$ and $C_{n} B_{m}$. This completes the proof.

REMARK 28. By induction, it can be proved that for all integers $m, n \leq 0,(7.4)$ holds. So for all integers $m, n,(7.4)$ is true. 
COROLlary 29. For all integers $m, n$, we have

$$
\begin{aligned}
& P_{n+m}=P_{n-2} P_{m}+P_{n-3} P_{m+1}+P_{n-4} P_{m-1}, \\
& E_{n+m}=E_{n-2} P_{m}+E_{n-3} P_{m+1}+E_{n-4} P_{m-1}, \\
& S_{n+m}=S_{n-2} P_{m}+S_{n-3} P_{m+1}+S_{n-4} P_{m-1}, \\
& A_{n+m}=A_{n-2} P_{m}+A_{n-3} P_{m+1}+A_{n-4} P_{m-1} .
\end{aligned}
$$

\section{References}

[1] Aarts, J., Fokkink, R., Krujitzer, G., Morphic Numbers, Nieuw Archief voor Wiskunde, 5(2), 56-58, 2001. www.nieuwarchief.nl/serie5/pdf/naw5-2001-02-1-056.pdf.

[2] Adams, W., Shanks, D., Strong Primality Tests That Are Not Sufficient, Mathematics of Computation, 39 (159), 255-300, 1982.

[3] Adegoke, K., Summation Identities Involving Padovan and Perrin Numbers, arXiv:1812.03241v2, 2019.

[4] Aküzüm Y., Deveci, Ö., Sylvester-Padovan-Jacobsthal-type Sequences, Maejo Int. J. Sci. Technol., 11(03), 236-248, 2017.

[5] Alsina, C., García-Roig, JL., On Plastic Numbers, Journal of Mathematics and Design, 1(1), 13-19, 2001.

[6] Ballantine, C., Merca, M., Padovan Numbers as Sums Over Partitions into Odd Parts, Journal of Inequalities and Applications, 2016:1, 2016. http://dx.doi.org/10.1186/s13660-015-0952-5.

[7] Basu, M., Das, M., Tribonacci Matrices and a New Coding Theory, Discrete Mathematics, Algorithms and Applications, 6 (1), 1450008, (17 pages), 2014.

[8] Boubellouta, K., Kerada, M., Some Identities and Generating Functions for Padovan Numbers, Tamap Journal of Mathematics and Statistics, Volume 2019, Article ID SI04, doi:10.29371/2019.16.SI04.

[9] Broadhurst, D.J., Kreimer, D., Association of Multiple Zeta Values with Positive Knots via Feynman Diagrams up to 9 Loops, arXiv:hep-th/9609128v3, 1996.

[10] Bród, D., A Note on a Graph Representation of Padovan Numbers, Int. J. Contemp. Math. Sciences, 5(42), 2065-2068, 2010.

[11] Bruce, I., A modified Tribonacci sequence, Fibonacci Quarterly, 22(3), 244-246, 1984.

[12] Catalani, M., Identities for Tribonacci-related sequences, arXiv:math/0209179, 2012.

[13] Cerda-Morales, G., On a Generalization of Tribonacci Quaternions, Mediterranean Journal of Mathematics 14:239, 1-12, 2017.

[14] Cerda-Morales, G., Identities for Third Order Jacobsthal Quaternions, Advances in Applied Clifford Algebras 27(2), 1043-1053, 2017.

[15] Cerda-Morales, G., New Identities for Padovan Numbers, arXiv:1904.05492, 2019.

[16] Cereceda, J. L., Determinantal Represantations for Generalized Fibonacci and Tribonacci Numbers, Int. J. Contemp. Math. Sciences. 9(6), 269-285, 2014.

[17] Choi, E., Modular Tribonacci Numbers by Matrix Method, Journal of the Korean Society of Mathematical Education Series B: Pure and Applied. Mathematics. 20(3), 207-221, 2013.

[18] Deveci, Ö., Padovan-Type Sequences via The Hurwitz Matrices, Karaelmas Fen ve Müh. Derg. 6(1):1-8, 2016.

[19] Deveci, Ö., Karaduman, E., On the Padovan p-numbers, Hacettepe Journal of Mathematics and Statistics, 46 (4), 579-592, 2017. 10.15672/HJMS.20174622760.

[20] Deveci, Ö., Shannon, A.G., Pell-Padovan-Circulant Sequences and Their Applications, Notes on Number Theory and Discrete Mathematics, 23(3), 100-114, 2017.

[21] Deveci, Ö., The Padovan-Circulant Sequences and Their Applications, Math. Reports, 20(70), 4, 401-416, 2018. 
[22] Dişkaya, O., Menken, Hamza, On the $(s, t)$-Padovan and $(s, t)$-Perrin Quaternions, J. Adv. Math. Stud. 12(2), 186-192, 2019 .

[23] Dişkaya, O., Menken, Hamza, On the Split $(s, t)$-Padovan and $(s, t)$-Perrin Quaternions, International Journal of Applied Mathematics and Informatics, 13, 25-28, 2019.

[24] Elia, M., Derived Sequences, The Tribonacci Recurrence and Cubic Forms, Fibonacci Quarterly, 39 (2), $107-115,2001$.

[25] Er, M. C., Sums of Fibonacci Numbers by Matrix Methods, Fibonacci Quarterly, 22(3), 204-207, 1984.

[26] Escott, E.B., ibid., v. 8, 63-64, 1901.

[27] Faisant, A., On The Padovan Sequence, arXiv:1905.07702v1, 2019.

[28] Gil, J.B., Weiner M.D., Zara, C., Complete Padovan Sequences in Finite Fields, Fibonacci Quarterly, 45(1), 64-75, 2007.

[29] Gogin N. D., Myllari A. A., The Fibonacci-Padovan sequence and MacWilliams Transform Matrices, Programing and Computer Software, 33(2), 74-79, 2007. Original Russian Text: published in Programmirovanie, 33(2), 2007.

[30] Gogin, N., Mylläri A., On the Average Growth Rate of Random Compositions of Fibonacci and Padovan Recurrences, in: V.P. Gerdt, E.W. Mayr, Ev.V. Vorozhtsov (Eds.), Computer Algebra in Scientific Computing, 11th International Workshop, CASC 2009, Kobe, Japan, September 13-17, in: Lecture Notes in Computer Science, vol. 5743, 240-246, 2009.

[31] Gogin, N., Mylläri, A., Padovan-like Sequences and Bell Polynomials, Mathematics and Computers in Simulation, 125, 168-177, 2016. https://doi.org/10.1016/j.matcom.2015.08.008

[32] Goy, T. Some Identities for Padovan Numbers via the Determinants of Toeplitz-Hessenberg Matrices / T. Goy // 30th Int. Conf. of the Jangjeon Mathematical Society. Pure and Applied Mathematics, 12-15 July 2017: Book of abstract. USTHB, Bab-Ezzouar, Algeria, 2017. - P. 242-244.

[33] Goy, T. Some Families of Identities for Padovan Numbers, Proceeding of Jangjeon Mathematical Society, 21(3), 413-419, 2018. http://dx.doi.org/10.17777/pjms2018.21.3.413

[34] Gunay, H, and Taskara, N., Some Properties of Padovan Quaternion, Asian-European Journal of Mathematics, 14(1), 2040017 (8 pages), 2020. DOI: 10.1142/S1793557120400173

[35] Halim, Y., Rabago, J.F.T., On the Solutions of a Second-Order Difference Equations in Terms of Generalized Padovan Sequences, Mathematica Slovaca, 68(3), 625-638, 2018. DOI: 10.1515/ms-2017-0130

[36] He., T., Liao, J.H.C., Shiue, P.J.S., Matrix Representation of Recursive Sequences of Order 3 and Its Applications, Journal of Mathematical Research with Applications, 38(3), 221-235, 2018. DOI:10.3770/j.issn:2095-2651.2018.03.001

[37] Howard F.T., Saidak, F., Zhou's Theory of Constructing Identities, Congress Numer. 200 (2010), $225-237$.

[38] Iliopoulos V., The Plastic Number and its Generalized Polynomial, Cogent Mathematics, 2:1, 1023123, 2015, DOI: $10.1080 / 23311835.2015 .1023123$.

[39] Irmak, N., On Factorials in Perrin and Padovan Sequences, Turkish Journal of Mathematics, 43, 2602-2609, 2019.

[40] Jarden, D., Recurring sequences: A collection of papers, Riveon Lematematika, Jerusalem, 1966.

[41] Kalman, D., Generalized Fibonacci Numbers By Matrix Methods, Fibonacci Quarterly, 20(1), 73-76, 1982.

[42] Kartal, M.Y., Gaussian Padovan and Gaussian Perrin Numbers and Properties of Them, Asian-European Journal of Mathematics, 12(6) (8 pages), 2040014, 2019. DOI: 10.1142/S1793557120400148.

[43] Kaygisiz, K., Bozkurt D., k-Generalized Order-k Perrin Number Presentation by Matrix Method, Ars Combinatoria, 105, 95-101, 2012.

[44] [Kaygisiz, K., Sahin A., Generalized Van der Laan and Perrin Polynomials, and Generalizations of Van der Laan and Perrin Numbers, Selçuk Journal of Applied Math., 14(1), 89-103,2013.

[45] Kaygisiz, K. and Sahin A., Calculating terms of associated polynomials of Perrin and Cordonnier numbers, Notes on Number Theory and Discrete Mathematics, 20(1)(2014),10-18.

[46] Khompungson, K., Rodjanadid, B., Sompong, S., Some Matrices in Term of Perrin and Padovan Sequences, Thai Journal of Mathematics, 17(3), 767-774, 2019. 
[47] Kiliç, E., Stanica, P., A Matrix Approach for General Higher Order Linear Recurrences, Bulletin of the Malaysian Mathematical Sciences Society, (2) 34(1), 51-67, 2011.

[48] Koshy, T., Fibonacci and Lucas Numbers with Applications. Wiley-Interscience. New York, 2001.

[49] Kovačić, B., Marohnić, L., Opačić, R., O Padovanovu nizu, Osjecki matematicki list 13, 1-19, 2013.

[50] Lin, P. Y., De Moivre-Type Identities For The Tribonacci Numbers, Fibonacci Quarterly, 26, $131-134,1988$.

[51] Lucas, E., Sur la recherché de grands nombres premiers, in A. F. Congrés du Clerment-Ferrand, pp. 61-68, 1876.

[52] Malo, E., ibid., v. 7, p. 281, p. 312, 1900.

[53] Marohnić, L., Strmečki T., Plastic Number: Construction and Applications, Advanced Research in Scientific Areas, 3(7), 1523-1528, 2012.

[54] Padovan sequence:

http://mathworld.wolfram.com/PadovanSequence.html

[55] Padovan sequence:

http://en.wikipedia.org/wiki/Padovan_sequence

[56] Perrin's Sequence:

https://www.mathpages.com/home/kmath345/kmath345.htm

[57] Proof of Generalized Little Theorem of Fermat:

https://www.mathpages.com/home/kmath346/kmath346.htm

[58] Padovan, R., Dom Hans van Der Laan and the Plastic Number. In: Williams K., Ostwald M. (eds) Architecture and Mathematics from Antiquity to the Future. Birkhäuser, Cham 407-419, 2015, https://doi.org/10.1007/978-3-319-001432_27. (First published as: Richard Padovan , "Dom Hans van Der Laan and the Plastic Number", pp. 181-193 in Nexus IV: Architecture and Mathematics, Kim Williams and Jose Francisco Rodrigues, eds. Fucecchio (Florence): Kim Williams Books, 2002. http://www.nexusjournal.com/conferences/N2002-Padovan.html)

[59] Padovan, R., Dom Hans van der Laan: Modern Primitive, Architectura and Natura Press, 1994.

[60] Perrin, R., Query 1484. L'Intermédiaire des Des Mathematiciens, 6, 76-77, 1899.

[61] Pethe, S., Some Identities for Tribonacci sequences, Fibonacci Quarterly, 26(2), 144-151, 1988.

[62] T. Piezas, A Tale of Four Constants, https://sites.google.com/site/tpiezas/0012.

[63] Prabowo, A., Nurshiami, S.R., Wijayanti, R., Sukono, F., The Padovan-like Sequence Raised From Padovan Q-Matrix, Proceeding of IORA International Conference on Operations Research 2017, Universitas Terbuka, Tangerang Selatan, Indonesia, 12 th October 2017.

[64] Şahin, A., On the Generalized Perrin and Cordonnier Matrices, Commun. Fac. Sci. Univ. Ank. Sér. A1 Math. Stat. 66(1), 242-253, 2017. DOI: 10.1501/Commua1_0000000793

[65] Scott, A., Delaney, T., Hoggatt Jr., V., The Tribonacci sequence, Fibonacci Quarterly, 15(3), $193-200,1977$.

[66] Seenukul, P., Netmanee, S., Panyakhun, T., Auiseekaen, R., Muangchan, S., Matrices which have similar properties to Padovan Q -Matrix and its generalized relations, SNRU Journal of Science and Technology 7(2), 90-94, 2015.

[67] Shannon, A., Tribonacci numbers and Pascal's pyramid, Fibonacci Quarterly, 15(3), pp. 268 and $275,1977$.

[68] A. G. Shannon, A.G., Anderson, P.G., Horadam A.F., Properties of Cordonnier, Perrin and Van der Laan numbers, International Journal of Mathematical Education in Science and Technology, 37(7), 825-831, 2006.

[69] Shannon, A.G., Horadam, A.F., Anderson, P.G., The Auxiliary Equation Associated With The Plastic Number, NNTDM $12(1), 1-12,2006$.

[70] Shtayat, J., Al-Kateeb, A., An Encoding-Decoding algorithm based on Padovan numbers, arXiv:1907.02007, 2019.

[71] Sloane, N.J.A., The on-line encyclopedia of integer sequences, http://oeis.org/

[72] Sompong, S., Wora-Ngon, N., Piranan, A., Wongkaentow, N., Some Matrices with Padovan Q-matrix Property, AIP Conference Proceedings 1905, 030035, 2017. https://doi.org/10.1063/1.5012181 
[73] Sokhuma, K., Padovan Q-Matrix and the Generalized Relations, Applied Mathematical Sciences, 7(56), 2777-2780, 2013.

[74] Sokhuma, K., Matrices Formula for Padovan and Perrin Sequences, Applied Mathematical Sciences, 7(142), 7093-7096, 2013.

[75] Soykan, Y., Summing Formulas For Generalized Tribonacci Numbers, Universal Journal of Mathematics and Applications, 3(1), 1-11, 2020. DOI: https://doi.org/10.32323/ujma.637876

[76] Soykan, Y., Simson Identity of Generalized m-step Fibonacci Numbers, Int. J. Adv. Appl. Math. and Mech. 7(2), 45-56, 2019 .

[77] Soykan, Y. Tribonacci and Tribonacci-Lucas Sedenions. Mathematics 7(1), 74, 2019.

[78] Spickerman, W., Binet's formula for the Tribonacci sequence, Fibonacci Quarterly, 20, 118-120, 1982.

[79] Spinadel, V.W., Buitrago, A.R., Towards van der Laan's Plastic Number in the Plane, Journal for Geometry and Graphics, $13(2), 163-175,2009$.

[80] Stewart, I., Tales of a Neglected Number, Mathematical Recreations, Scientific American, 274(6), 102-103, 1996.

[81] Taş, Sait., Karaduman, Erdal., The Padovan Sequences in Finite Groups, Chiang Mai Journal of Science 41(2), 456-462, 2014. DOI: $10.14456 /$ mijst.2014.21

[82] Taşcı, D., and Acar, H., Gaussian Padovan and Gaussian Pell-Padovan Numbers, Commun. Fac. Sci. Ank. Ser. A1 Math. Stat., 67(2), 82-88, 2018.

[83] Tascı, D., Padovan and Pell-Padovan Quaternions, Journal of Science and Arts, 1(42), 125-132, 2018.

[84] Taşyurdu, Yasemin., Akpınar, Ayşe., Padovan and Pell-Padovan Octonions, Proceedings of International Conference on Mathematics and Mathematics Education (ICMME 2019), Turk. J. Math. Comput. Sci. 11(Special Issue) 114-122, 2019.

[85] Van Der Laan, H., Le Nombre Plastique; quinze Leçons sur l'Ordonnance architectonique, Brill, Leiden 1960.

[86] Vieira, R.P.M., Alves, F.R.V., A Sequência de Padovan e o número plástico: uma análise prévia e a priori (The Padovan Sequence and the plastic number: a prior analysis and a priori),Res., Soc. Dev. 8(8), 2019, http://dx.doi.org/10.33448/rsdv8i8.1212.

[87] Voet, C., The Poetics of Order: Dom Hans Van der Laan's Architectonic Space, Architectural Research Quarterly, 16(2), 137-154, 2012. doi:10.1017/S1359135512000450.

[88] Yalavigi, C. C., Properties of Tribonacci numbers, Fibonacci Quarterly, 10(3), 231-246, 1972.

[89] Yaşar, M., Hessenberg Matrices and Padovan Numbers, AIP Conference Proceedings 1863, 310005, 2017, https://doi.org/10.1063/1.4992481

[90] Yilmaz, F., Bozkurt D., Hessenberg Matrices and the Pell and Perrin Numbers, Journal of Number Theory, 131, 1390$1396,2011$.

[91] Yilmaz F., Bozkurt, D., Some Properties of Padovan Sequence by Matrix Methods, Ars Combin. 104, 149-160, 2012.

[92] Yilmaz, N., Taskara, N., Matrix Sequences in Terms of Padovan and Perrin Numbers, Journal of Applied Mathematics, Volume 2013, 2013, Article ID 941673, 7 pages, http://dx.doi.org/10.1155/2013/941673.

[93] Yilmaz N., Taskara, N., Binomial Transforms of the Padovan and Perrin Matrix Sequences, Abstract and Applied Analysis, Volume 2013 Article ID 497418, 2013.

[94] Yilmaz, N., Taskara, N., On the Negatively Subscripted Padovan and Perrin Matrix Sequences, Communications in Mathematics and Applications, 5(2) (2014), 59-72, 2014.

[95] Yilmaz, N., Taskara, N., Tribonacci and Tribonacci-Lucas Numbers via the Determinants of Special Matrices, Applied Mathematical Sciences, 8(39), 1947-1955, 2014.

[96] Zangana, D.O.M., Öteleş, A., Padovan Numbers by the Permanents of a Certain Complex Pentadiagonal Matrix, Journal of Garmian University, 5(2), 330-338, 2018. 